\title{
Effect of Salt Identity on the Phase Diagram for a Globular Protein in Aqueous Electrolyte Solution
}

\author{
Mathias Boström, ${ }^{\dagger}$ Frederico W. Tavares, ${ }^{*}$, Barry W. Ninham, ${ }^{\S}$ and John M. Prausnitz ${ }^{\perp}$ \\ Department of Physics, Chemistry and Biology, Linköping University, SE-581 83, Linköping, Sweden, \\ Escola de Química, Universidade Federal do Rio de Janeiro, Caixa Postal 68542, CEP 21949-900, \\ Rio de Janeiro, RJ, Brazil, Department of Applied Mathematics, Australian National University, \\ Canberra, Australia, Department of Chemical Engineering, University of California, \\ Berkeley, California 94720-1462, and Chemical Sciences Division, \\ Lawrence Berkeley National Laboratory, Berkeley, California 94720
}

Received: February 24, 2006; In Final Form: July 4, 2006

\begin{abstract}
Monte Carlo simulations are used to establish the potential of mean force between two globular proteins in an aqueous electrolyte solution. This potential includes nonelectrostatic contributions arising from dispersion forces first, between the globular proteins, and second, between ions in solution and between each ion and the globular protein. These latter contributions are missing from standard models. The potential of mean force, obtained from simulation, is fitted to an analytic equation. Using our analytic potential of mean force and Barker-Henderson perturbation theory, we obtain phase diagrams for lysozyme solutions that include stable and metastable fluid-fluid and solid-fluid phases when the electrolyte is $0.2 \mathrm{M} \mathrm{NaSCN}$ or $\mathrm{NaI}$ or $\mathrm{NaCl}$. The nature of the electrolyte has a significant effect on the phase diagram.
\end{abstract}

\section{Introduction}

The phase behavior of colloidal solutions is relevant to a variety of technical applications. Examples include paints, inks, coatings, and high-tech materials for optical, structural, and medical purposes. In addition, for biotechnology it is important to understand the phase behavior of aqueous protein solutions. ${ }^{1}$ However, previously published models cannot account for the effect of salt identity on the phase diagram; they do not explain the classical Hofmeister problem: why, for example, different concentrations of $\mathrm{NaCl}$ and $\mathrm{NaSCN}$ are needed to precipitate hen-egg-white protein from aqueous solution. Contrary to experiment, previous models predict that the required concentration for $\mathrm{NaCl}$ should be the same as that for NaSCN. In this work we show that inclusion of ion-specific dispersion potentials, ${ }^{2,3}$ missing from standard models of colloidal interactions, gives ion-specific phase diagrams that agree with experiment. Inclusion of these nonelectrostatic dispersion potentials has previously been shown to explain ion specificity in proteinprotein interactions when the salt concentration exceeds (about) $0.1 \mathrm{M}^{3}$

Anderson and Lekkerkerker ${ }^{4}$ (and others) have indicated that colloidal solutions display intriguing phase transitions spanning fluid-fluid, fluid-solid, and solid-solid phases. Ishimoto and Tanaka ${ }^{5}$ were the first to report the existence of temperatureinduced liquid-liquid-phase separation in aqueous lysozymeelectrolyte solution. Taratuta et al. $^{6}$ showed that for such solutions, the cloud-point temperature depends on the choice of anion in the electrolyte solution.

We are here interested in the phase behavior of lysozyme solutions containing any one of three different monovalent salts,

* Address correspondence to this author.

$\dagger$ Linköping University.

$\doteqdot$ Universidade Federal do Rio de Janeiro.

$\S$ Australian National University.

${ }^{\perp}$ University of California and Lawrence Berkeley National Laboratory. all at the same concentration. First-order perturbation theory, combined with a realistic potential of mean force between two protein particles, allows prediction of the rich phase behavior. Our calculations require no adjustable fitting parameters.

Aqueous protein solutions often exist for a long time under metastable conditions. ${ }^{7}$ Existence of a metastable region in a phase diagram may be important because it influences the rate of phase transformations, e.g., crystallization. ${ }^{4,8,9}$ The literature provides numerous articles that discuss phase diagrams of colloidal and similar solutions; see, e.g., pertinent references in Tavares and Prausnitz. ${ }^{10}$

Most articles in the literature present phase behavior in a temperature-density plot obtained from molecular simulations. However, for applications, especially for optimization in product and process design, it is preferable to use perturbation theory using an analytical potential of mean force acting between two aqueous proteins. We first describe how we use Monte Carlo simulation to obtain the potential of mean force between two lysozyme particles. After fitting this potential to an analytic function, we then use first-order perturbation theory to predict the phase behavior of aqueous lysozyme solutions that contain a specific electrolyte. (We have earlier shown that first- and second-order perturbation theories are nearly equivalent for calculating phase diagrams for this kind of potential. ${ }^{10}$ )

\section{Potential of Mean Force for Lysozyme}

We calculated the potential of mean force between two macroions that mimic lysozyme at $\mathrm{pH} \sim 4.3$; the macroions were represented by hard spheres with effective diameter $\sigma=33 \AA$, bearing a charge of $+10 \mathrm{e}^{0} .{ }^{11}$ In our calculations we include not only conventional electrostatic, hard-sphere, and macroionmacroion dispersion (Hamaker) interactions, but also ionspecific ion-ion and ion-macroion dispersion interactions. The dispersion interactions are the same as those used recently by Tavares et al. ${ }^{3}$ The ion-ion dispersion potentials are given by 
TABLE 1: Dispersion Coefficients for Ion-Ion and for Ion-Macroion Interactions from Tavares et al. ${ }^{3 a}$ Except the Dispersion Coefficients for $\mathrm{SCN}^{-}$from Ref $3 \mathrm{C}$

\begin{tabular}{lccc}
\hline ion & $\begin{array}{c}B_{\text {ion-ion }} \\
\left(10^{-80} \mathrm{~J} \mathrm{~m}^{6}\right)\end{array}$ & $\begin{array}{c}B_{\text {ion- } \mathrm{Na}^{+}} \\
\left(10^{-80} \mathrm{~J} \mathrm{~m}^{6}\right)\end{array}$ & $\begin{array}{c}B_{\text {ion-macroion }} \\
\left(10^{-50} \mathrm{~J} \mathrm{~m}^{3}\right)\end{array}$ \\
\hline $\mathrm{Na}^{+}$ & -5.4 & -5.4 & -0.45 \\
$\mathrm{Cl}^{-}$ & -330.3 & -26.9 & -3.57 \\
$\mathrm{I}^{-}$ & -965.6 & -40.9 & -4.44 \\
$\mathrm{SCN}^{-}$ & -1930 & -82.5 & -10.0
\end{tabular}

$U_{\text {ion-ion }}=B_{\text {ion-ion }} /(r){ }^{6}$ for ion - macroion, the potentials are $U_{\text {ion-macroion }}=B_{\text {ion-macroion }} /\left(r-r_{\mathrm{p}}\right)^{3}$, where $\mathrm{r}$ is the center-tocenter distance and $r_{\mathrm{p}}=\sigma / 2$ is the radius of the protein. The dispersion coefficients $B_{\text {ion-ion }}$ depend on the ions and $B_{\text {ion-macroion }}$ depend on the ion and on the protein. The dispersion potentials are ion-specific because they depend on the polarizabilities of the ions and macroion. Following our previous work, ${ }^{3 a}$ Table 1 gives dispersion coefficients. For $\mathrm{SCN}^{-}$, the dispersion coefficients are taken from ref $3 \mathrm{~b}$.

Standard canonical Monte Carlo simulation is used. During each run, the two macroion particles are fixed at a given separation along the box diagonal, while the small ions are free to move throughout the box. The Ewald-sum method is applied to account for long-range electrostatic interactions. The large size of the protein requires that we perform simulations larger than those used by Tavares et al..$^{3 a}$ to account for the large number of counterions physisorbed on the protein surface whenever the anion is highly polarizable. The cubic simulation box contains two proteins and 600 small ions; all of the latter have the same diameter ( $4 \AA$ ). We maintain overall charge neutrality. The volume of the box is adjusted to give the desired ionic strength, here fixed at $0.2 \mathrm{M}$. The box size is about 1 order of magnitude larger than the Debye screening length. For each macroion-macroion configuration we performed $60 \times 10^{6}$ trial moves for equilibration and $120 \times 10^{6}$ for production. Details of the simulation are similar to those given by Tavares et $\mathrm{al}^{3}$

The dispersion potential of the ion-macroion is only modestly long range and the important contribution is for ions close to a macroion, especially in the simulations presented here, where the salt concentration is $0.2 \mathrm{M}$, i.e., the simulation box is very large compared to ion and macroion sizes. Boström et al. ${ }^{3 \mathrm{c}}$ show a comparison of concentration profiles near a macroion for different salts obtained from numerical solutions of the nonlinear Poisson-Boltzmann equation and the Ornstein-Zernike equation with Monte Carlo simulation data. The simulations in ref $3 \mathrm{c}$ are similar to those used in this paper. Results obtained from numerical calculations are in very good agreement with those from Monte Carlo simulations, showing that the calculations and simulations represent similar thermodynamic systems.

Figure 1 shows the ion-specific potential of mean force in three different monovalent salt solutions $(0.2 \mathrm{M} \mathrm{NaCl}, 0.2 \mathrm{M}$ $\mathrm{NaI}$, and 0.2 M NaSCN). In addition to classical ion-ion, ionmacroion, and macroion-macroion electrostatic interactions, these potentials include contributions from ion-ion and ionprotein dispersion forces. They become more attractive from $\mathrm{NaCl}$ to $\mathrm{NaI}$ to $\mathrm{NaSCN}$, consistent with the experimental observation that $\mathrm{NaSCN}$ is much more effective in precipitating hen-egg-white protein than the other salts.

In our phase-diagram calculations, we used the potential of mean force calculated numerically from our simulations. The resulting potentials were fitted to a fifth-order polynomial in $r / \sigma$, as expressed in Table 2; results are shown in Figure 1.

The total potential of mean force is

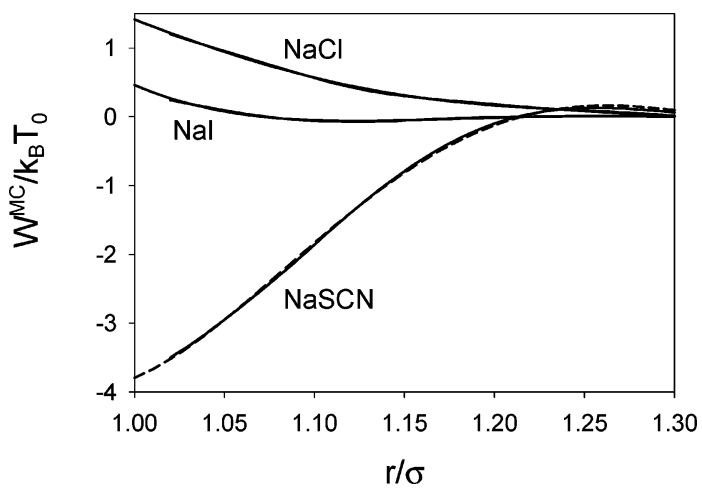

Figure 1. Effect of electrolyte on the protein-protein potential of mean force $\left(W^{\mathrm{MC}}\right)$ at $298 \mathrm{~K}$, obtained from Monte Carlo simulations, when that salt concentration is $0.2 \mathrm{M}$. These calculations exclude the direct lysozyme-lysozyme Hamaker dispersion interaction. The lysozyme charge is $+10 \mathrm{e}^{0}$ (for $\mathrm{pH} \sim 4.3$ ) and the lysozyme hard-sphere diameter, $\sigma$, is $3.3 \mathrm{~nm}$. The ion-ion and ion-lysozyme dispersion parameters are given in Table 1. All (small) ion diameters are $4 \AA$ A Here $T_{0}=298$ $\mathrm{K}$ and $k_{\mathrm{B}}$ is Boltzmann's constant.

TABLE 2: Potential of Mean Force Obtained from Monte Carlo Simulation ${ }^{a}$

\begin{tabular}{lccccc}
\hline \multicolumn{1}{c}{ salt } & $y_{0}$ & \multicolumn{1}{c}{$a$} & \multicolumn{1}{c}{$b$} & \multicolumn{1}{c}{$c$} & \multicolumn{1}{c}{$d$} \\
\hline $\mathrm{NaCl}$ & -224.32 & 819.4 & -1027.56 & 471.879 & -37.9813 \\
$\mathrm{NaI}$ & 494.621 & -1505.25 & 1623.79 & -654.982 & 42.2869 \\
$\mathrm{NaSCN}$ & 1623 & -5274 & 6008 & -2537 & 175.9
\end{tabular}

${ }^{a}$ The fitted potential is $W^{\mathrm{MC}} /\left(k_{\mathrm{B}} T_{0}\right)=y_{0}+a s+b s^{2}+c s^{3}+d s^{5}$, where $s=r / \sigma$ and $1<s<1.3$. The coefficients $y_{0}, a, b, c$, and $d$ are obtained by a nonweighted least-squares minimization method. These expressions correlate the Monte Carlo data with high precision.

$$
W^{\text {total }}=W^{\mathrm{HS}}+W^{\text {Hamaker }}+W^{\mathrm{MC}}
$$

where the superscript MC stands for Monte Carlo and HS for hard-sphere; for each salt the total potential of mean force is obtained by combining the result from Monte Carlo simulations with a hard-sphere potential:

$$
W^{\mathrm{HS}}=\left\{\begin{array}{l}
\infty \text { for } r \leq \sigma \\
0 \text { for } r>\sigma
\end{array}\right.
$$

and the well-known attractive Hamaker dispersion interaction: 12

$$
\begin{aligned}
& W^{\text {Hamaker }}= \\
& \left\{\begin{array}{l}
\frac{-H}{12}\left(\frac{\sigma^{2}}{(\sigma+2 \kappa)^{2}-\sigma^{2}}+\frac{\sigma^{2}}{(\sigma+2 \kappa)^{2}}+2 \ln \left(1-\frac{\sigma^{2}}{(\sigma+2 \kappa)^{2}}\right)\right) \text { for } r \leq \sigma+2 \kappa \\
\frac{-H}{12}\left(\frac{\sigma^{2}}{r^{2}-\sigma^{2}}+\frac{\sigma^{2}}{r^{2}}+2 \ln \left(1-\frac{\sigma^{2}}{r^{2}}\right)\right) \text { for } r>\sigma+2 \kappa
\end{array}\right.
\end{aligned}
$$

where $H$ is the Hamaker constant, here $10 k_{\mathrm{B}} T_{0}$, and $\kappa$ is the protein hydration-layer thickness, $1.5 \AA .{ }^{12} T_{0}$ is $298 \mathrm{~K}$ and $k_{\mathrm{B}}$ is Boltzmann's constant.

The potential of mean force used here is only proper for temperatures not far removed from $298 \mathrm{~K}$. Nevertheless, from DLVO theory, the potential of mean force depends on $\epsilon T$, where $\epsilon$ is the dielectric constant of water. The dielectric constant of water is a function of $T$ (CRC Handbook of Chemistry and Physics, 3rd electronic edition). Upon plotting $\epsilon$ and $\epsilon T^{*}$ as a function of reduced temperature $\left(T^{*}=T / 298 \mathrm{~K}\right)$ in Figure 2, we see that $\epsilon T^{*}$ remains close to 78 when the reduced temperature varies from 0.6 to 2.0. Consequently, our potential of mean force $W^{\text {total }}$ is considered to be independent of temperature for the range of $0.6<T^{*}<2.0$. Our potential of 


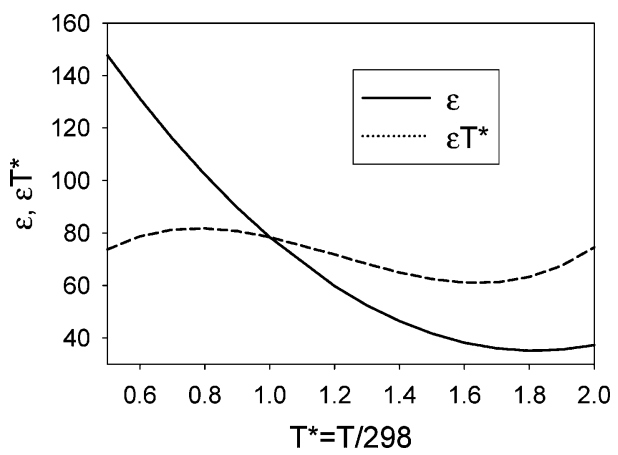

Figure 2. Dielectric constant of water $\epsilon$ and $\epsilon T^{*}$ as a function of reduced temperature. While $\epsilon$ changes appreciably with $T$, the product $\epsilon T^{*}$ is nearly constant.

mean force is consistent with calculations of Striolo et al. ${ }^{13}$ for charged dipolar colloids.

\section{Phase Diagrams for Lysozyme Solutions}

Long-range attractive interactions between two molecules have a strong influence on fluid-fluid equilibria. As shown by numerous authors, the range of the attractive interaction affects the stability of a fluid-fluid transition (see references in Tavares and Prausnitz ${ }^{10}$ ). When the interaction potential becomes shortranged, the stable fluid-fluid phase transition disappears and only the solid-fluid coexistence curve is thermodynamically stable. The range of attraction in the potential cannot be changed for ordinary fluids, but it can be adjusted in aqueous protein systems by adding, for example, a nonadsorbing polymer. Notably, as shown here, consistent with experiment, it can also be adjusted by changing the specific electrolyte.

We obtain thermodynamic properties and phase diagrams using pertinent first-order perturbation theories, one for the fluid and another for the solid. The potential of mean force, eq 1, is assumed to be independent of temperature and valid in all range of protein concentration. Strictly speaking, the resulting potential is an effective two-body potential, applicable only to a dilute protein solution. However, on the basis of computer simulations for polar and nonpolar ionic colloids, several authors ${ }^{14-16}$ have showed that the effect of neglecting many-body contributions is relatively small. Therefore, we use here the assumption of pairwise additivity to calculate the potential of mean force at all protein concentrations. The total Helmholtz energy of the system, $A$, is the sum of the hard-sphere and perturbation contributions:

$$
\frac{A}{N k_{\mathrm{B}} T}=\frac{A^{\mathrm{HS}}}{N k_{\mathrm{B}} T}+\frac{A^{\mathrm{P}}}{N k_{\mathrm{B}} T}
$$

where $A^{\mathrm{HS}}$ is the total Helmholtz energy for the hard-sphere fluid (reference system) and $A^{\mathrm{P}}$ is the perturbation contribution. $N$ is the number of molecules. The equation of state is obtained by differentiating the Helmholtz energy with respect to the packing fraction $\eta$ :

$$
Z=\eta\left(\frac{\partial\left(A / N k_{\mathrm{B}} T\right)}{\partial \eta}\right)=Z^{\mathrm{HS}}+Z^{\mathrm{P}}
$$

where $Z=p /\left(\rho k_{\mathrm{B}} T\right)$ is the compressibility factor of the system; $p$ is the pressure, and $\rho$ is the density. The packing fraction $\eta$ $=\left(\pi \rho \sigma^{3}\right) / 6$, and $\rho \sigma^{3}$ is the reduced density. $Z^{\mathrm{HS}}$ and $Z^{\mathrm{P}}$ are compressibility-factor contributions, one from the hard-sphere reference system and the other from the perturbation. Here, the

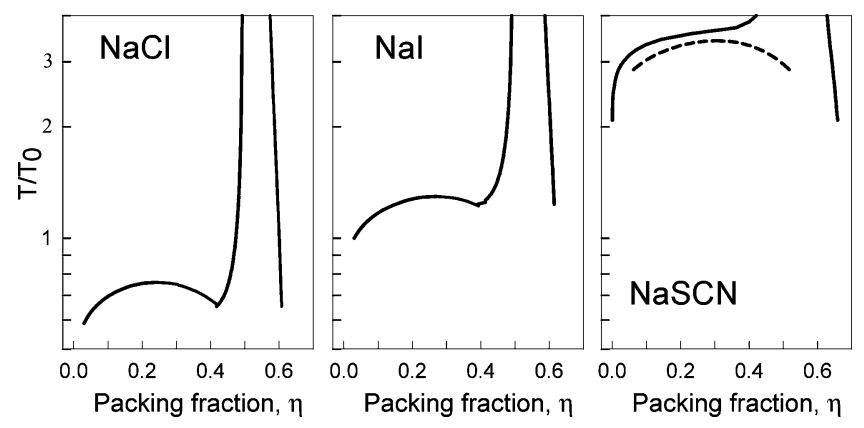

Figure 3. Phase diagrams calculated from first-order perturbation theory for three aqueous lysozyme solutions containing $\mathrm{NaCl}$, $\mathrm{NaI}$, or $\mathrm{NaSCN}$. For all cases, salt concentration is $0.2 \mathrm{M}$. Stable fluid-fluid and fluid-solid transitions appear for solutions containing $\mathrm{NaCl}$ and $\mathrm{NaI}$ (solid lines). When the electrolyte is $\mathrm{NaSCN}$, the stable transition is fluid-solid for all packing fractions. The dashed line represents metastable fluid-fluid transition when $\mathrm{NaSCN}$ is the electrolyte. Here $T_{0}$ is $298 \mathrm{~K}$.

perturbation part of the potential is the sum $W^{\text {Hamaker }}+W^{\mathrm{MC}}$ as indicated in eq 1 .

The chemical potential of the protein, $\mu$, is obtained from

$$
\frac{\mu}{k_{\mathrm{B}} T}=\frac{A}{N k_{\mathrm{B}} T}+Z=\frac{\mu^{\mathrm{HS}}}{k_{\mathrm{B}} T}+\frac{\mu^{\mathrm{P}}}{k_{\mathrm{B}} T}
$$

where $\mu^{\mathrm{HS}}$ and $\mu^{\mathrm{P}}$ are respectively the hard-sphere and perturbation contributions. For the hard-sphere contribution, we use the Carnahan-Starling equation for the fluid and the Velasco et al. model for the solid (refs 10 and 13, and references therein).

We use first-order Barker-Henderson perturbation theory to calculate the perturbation contribution to the Helmholtz energy for the fluid and for the solid as discussed previously by Tavares and Prausnitz. ${ }^{10}$

For calculating the phase diagram at a fixed temperature, either for solid-fluid or for fluid-fluid coexistence, we use the conventional equations for phase equilibria:

$$
\begin{aligned}
& \mu^{\prime}=\mu^{\prime \prime} \\
& p^{\prime}=p^{\prime \prime}
\end{aligned}
$$

where superscripts prime and double prime "' refer to phases.

In the isothermal equations for phase equilibria, chemical potential $\mu$ and pressure $p$ are functions of density (or packing fraction). There are two unknowns: the density of the prime phase ' and the density of the double prime phase". These densities of the coexisting phases are obtained by simultaneous solution of eqs 7 and 8 .

Using first-order perturbation theory and the potential of mean force described above, we obtain the phase diagrams shown in Figure 3. The three different electrolytes give significantly different results. At low packing fractions, the systems with 0.2 $\mathrm{M} \mathrm{NaCl}$ and $0.2 \mathrm{M} \mathrm{NaI}$ show stable fluid-fluid phase transitions. However, for liquid-liquid equilibria, sodium iodide exhibits a critical temperature higher than that for sodium chloride. At higher packing fractions, these electrolytes give stable fluid-solid-phase transition. However, in 0.2 M NaSCN with its large attractive potential of mean force, the fluid-fluid phase transition is metastable, i.e., at a fixed temperature smaller than the fluid-fluid critical point, eqs 7 and 8 give two possibilities for phase coexistence, solid-fluid and fluid-fluid. However, the chemical potentials obtained for solid-fluid calculations are smaller than those for fluid-fluid. Therefore, the solid-fluid equilibrium is stable when compared with fluidfluid equilibrium. At all packing fractions this system shows a 
stable fluid-solid-phase transition, consistent with laboratory use of $0.2 \mathrm{M}$ NaSCN for precipitating lysozyme and consistent with light-scattering data that indicate lysozyme-lysozyme interactions that are more attractive in aqueous $\mathrm{NaSCN}$ than in aqueous $\mathrm{NaCl}{ }^{12,17}$

The phase diagrams presented here are necessarily approximate because they are based on calculations that make two major assumptions: first, the dispersion part of our potential of mean force is independent of temperature and second, our potential of mean force is independent of protein concentration. This latter assumption, similar to that in the van der Waals equation, is necessary for our purpose: to present a simple analytic theory for calculating the phase diagram, i.e., to establish a reasonable, simplified theoretical method that, in essence, is similar to a van der Waals-type theory for ordinary fluids. The dispersion contribution to our potential of mean force is new, generated by suitable molecular physics, evaluated numerically by computer calculations, and then fitted to a powerseries equation to facilitate analytic calculation of the phase diagram using first-order perturbed hard-sphere theory. The physically significant parameters are the polarizabilities in water of the macro- and microions. We estimate these polarizabilities from experimental data at or near our reference temperature $T_{0}$ $=298 \mathrm{~K}$. Because we do not have the necessary information to say anything about the effect of temperature on these polarizabilities, our phase-diagram calculations are unavoidably based on a potential of mean force whose dispersion contributions are assumed to be independent of temperature. Thus, in the calculation of aqueous-protein phase diagrams, valid for temperatures near $298 \mathrm{~K}$, our work provides one (surely not the last) step forward, because in previous studies of such phase diagrams, no attention at all was given to the dispersion effects discussed here.

\section{Conclusions}

Calculated phase diagrams for aqueous lysozyme solutions with three different electrolytes show, in accord with experiment, that interactions between a pair of lysozyme particles become more attractive when the $0.2 \mathrm{M}$ electrolyte changes from $\mathrm{NaCl}$ to $\mathrm{NaI}$ to $\mathrm{NaSCN}$. Our calculations take into account that, at constant ionic strength, phase-transition equilibria depend on the choice of salt. This dependence can be traced to differences in adsorption of ions to the macroion surface caused by ionion and ion-macroion dispersion potentials.

Acknowledgment. For financial support M.B. thanks the Swedish Research Council, J.M.P. thanks the Office for Basic Sciences of the U.S. Department of Energy, and F.W.T. thanks CNPq and FAPERJ.

\section{References and Notes}

(1) Evans, D. F.; Wennerström, H. The colloidal domain: where physics, chemistry, biology, and technology meet, 2nd ed.; Wiley-VCH: New York, 1999.

(2) Boström, M.; Williams, D. R. M.; Ninham, B. W. Phys. Rev. Lett. 2001, 87, 168103/1.

(3) (a) Tavares, F. W.; Bratko, D.; Blanch, H.; Prausnitz, J. M. J. Phys. Chem. $B$ 2004, 108, 9228. (b) Boström, M.; Williams, D. R. M.; Ninham, B. W. Biophys. J. 2003, 85, 686. (c) Boström, M.; Tavares, F. W.; Bratko, D.; Ninham, B. W. J. Phys. Chem. B 2005, 109, 24489.

(4) Anderson, V. J.; Lekkerkerker, H. N. W. Nature 2002, 416, 811.

(5) Ishimoto, C.; Tanaka, T. Phys. Rev. Lett. 1977, 39, 474.

(6) Taratuta, V. G.; Holschenbach, A.; Thurston, G. M.; Blankschtein, D.; Benedek, G. B. J. Phys. Chem. 1990, 94, 2140.

(7) Debenedetti, P. G. Metastable Liquids: Concepts and Principles; Princeton University Press: Princeton, NJ, 1996.

(8) Renth, F.; Poon, W. C. K.; Evans, R. M. L. Phys. Rev. E 2001, 64 031402 .

(9) Evans, R. M. L.; Poon, W. C. K.; Renth, F. Phys. Rev. E 2001, 64, 031403

(10) Tavares, F. W.; Prausnitz, J. M. Colloid Polym. Sci. 2004, 282,

(11) (a) Teske, C. A.; Blanch, H. W.; Prausnitz, J. M. Fluid Phase Equilib. 2004, 219. 139. (b) Kuehner, D. E.; Prausnitz, J. M.; Fergg, F.; Wernick, M.; Blanch, H. W.; Engmann, J. J. Phys. Chem. B 1999, 103 , $1368-1374$

(12) Curtis, R. A.; Ulrich, J.; Montaser, A.; Prausnitz, J. M.; Blanch, H. W. Biotechnol. Bioeng. 2002, 79, 367.

(13) Striolo, A.; Tavares, F. W.; Bratko, D.; Blanch, H. W.; Prausnitz, J. M. Phys. Chem. Chem. Phys. 2003, 5, 4851.

(14) Wu, J. Z.; Bratko, D.; Blanch, H. W.; Prausnitz, J. M. J. Chem. Phys. 2000, 113, 3360.

(15) Linse, P. J. Phys.: Condens. Matter 2002, 14, 13449.

(16) Tavares, F. W.; Bratko, D.; Striolo, A.; Blanch, H. W.; Prausnitz, J. M. J. Chem. Phys. 2004, 120, 9859.

(17) Boström, M.; Tavares, F. W.; Finet, S.; Skouri-Panet, F.; Tardieu, A.; Ninham, B. W. Biophys. Chem. 2005, 117, 217. 